\title{
Assessment of Surgical Outcomes in Children and Adult Ischemic Moyamoya Disease and Its Relationship with the Pre-infarction Cerebral Perfusion Status
}

\author{
Peijiong WANG ${ }^{1}$, Husule CAI ${ }^{1}$, Rutao LUO², Xingju LIU1', Dong ZHANG¹, Yan ZHANG ${ }^{1}$ \\ ${ }^{1}$ Capital Medical University, Beijing Tiantan Hospital, Department of Neurosurgery, Beijing, China \\ ${ }^{2}$ Capital Medical University, National Center for Children's Health, Beijing Children's Hospital, Department of Neurosurgery, Beijing, China
}

Corresponding author: Yan ZHANG yanzhang135@163.com

\section{ABSTRACT}

AIM: To clarify perfusion differences, and to determine whether children and adults respond similarly to surgical prevention and how brain perfusion stages before surgery predict outcomes in ischaemic moyamoya disease (MMD) in children and adults.

MATERIAL and METHODS: A total of 355 patients with ischaemic MMD, including 74 children and 281 adults, were enrolled in the study. Computerized tomography perfusion (CTP) scans were used to identify the perfusion status according to a novel staging system of the pre-infarction period. The perfusion status of each hemisphere between the children and adult groups was analysed. The modified Rankin scale was used during long-term follow-up as an indicator of clinical outcomes.

RESULTS: The proportions of stages 0 and IV in adults were significantly higher than those in children ( $p=0.09$ and $p=0.003$, respectively). Stage III was more common in the children's group $(\mathrm{p}=0.001)$. The stroke data showed an increasing tendency in the infarction rate from stages I to IV. Both groups in stage 0 and in the early stages had a similar highly improved ratio after surgery; the children, however, achieved significantly better clinical outcomes in stage III and late stages.

CONCLUSION: There are differences in the perfusion status between child and adult patients with MMD. The pre-infarction staging system is associated with MMD-related stroke to some extent. Children have a greater chance for improvement than adults in stage III and later stages.

KEYWORDS: Moyamoya disease, Cerebral blood flow, Computerised tomography, Treatment outcome

ABBREVIATIONS: CB: Combined bypass, Cl: Confidence interval, CT: Computerised tomography, CTP: Computerised tomography perfusion image, DB: Direct bypass, IB: Indirect bypass, DSA: Digital subtraction angiography, m: Month, MMD: Moyamoya disease, mRS: Modified Rankin scale, MRA: Magnetic resonance angiography, n: Number, rCBV: Regional cerebral blood volume, rCBF: Regional cerebral blood flow, rTIA: Regional transient ischaemic attack, rTTP: Regional time to peak, rMTT: Regional mean transit time

\section{INTRODUCTION}

$\mathrm{M}$ oyamoya disease (MMD) is a chronic cerebral vascular disease characterised by the progressive occlusion of either or both the terminal internal carotid artery and other large intracranial arteries and the formation of net-like collateral arteries $(11,21,22)$. The majority of patients with MMD develop cerebral ischaemic symptoms, including cerebral infarctions and transient ischaemic attacks (TIAs), intracranial haemorrhage and other symptoms such as headache and epilepsy $(2,22)$.
Peijiong WANG (1) : 0000-0002-3364-4035

Husule CAI (1D): 0000-0002-2421-4586

Rutao LUO (1) : 0000-0002-9510-0420
Xingju LIU (1) : 0000-0003-2009-908X

Dong ZHANG (1) : 0000-0002-0801-4971

Yan ZHANG (1) : 0000-0003-1634-4484 
A few staging systems for ischaemic MMD have been developed to assess the status of cerebral perfusions, which is associated with clinical symptoms and postprocedural outcomes $(4,24)$. In a previous study, Gao et al. proposed a computerised tomography perfusion (CTP) image scale system for the pre-infarction stages to classify the cerebral hypoperfusion status (19). Furthermore, Yin et al. demonstrated the association between this staging system and clinical outcomes of patients with MMD and illustrated how patients with a different cerebral perfusion status might achieve different post-procedure outcomes (25). Some reports also showed children with ischaemic MMD achieving better post-procedure outcomes than adults $(5,24)$, but the differences in cerebral perfusion of ischaemic MMD between adults and children are not fully investigated yet. Moreover, MMD is a heterogeneous disease $(5,6,10,14,22)$ and whether there are differences in post-procedure outcomes between adults and children for each cerebral perfusion status level remains unknown.

In this study, we used the CTP scale system for the preinfarction period to explore the differences in cerebral perfusion before surgery between adults and children, and we investigated the possible relationship between the cerebralhypoperfusion staging system scale and clinical symptoms and the association between this staging system and surgical outcomes.

\section{MATERIAL and METHODS}

This was a retrospective, single-centre study on MMD. The study was approved by the Beijing Tiantan Hospital board and was supported mainly by a major scientific project of the $13^{\text {th }}$ National 5-year Plan and the Program of the National Natural Science Foundation of China (81870833). The data were collected in Beijing Tiantan Hospital from June 2011 to November 2017, and all enrolled patients signed a consent form. Information gathered in this study included clinical records, radiology data and follow-up status. MMD was diagnosed according to the guidelines for MMD through DSA/MRA examination (criteria of the Research Committee on Spontaneous Occlusion of the Circle of Willis, 2012). All patients had ischaemic symptoms, including stroke, seizure, TIA and headache before surgery. Patients were excluded from this study if their follow-up period was less than 12 months. Patients were divided into the children's group (age < 18 years old) and adult group (age $\geq 18$ years old).

\section{CTP Examination}

All patients received a CTP examination at most six months before revascularisation surgery. During the examination, a regular computerised tomography (CT) scan (GE-Healthcare Discovery CT 750HD) was conducted, followed by a bolus injection of $40-\mathrm{ml}$ iobitridol $(350 \mathrm{mg} / \mathrm{ml}$, Xenetix; Guerbet Aulnay-sous-Bois, France) into the antecubital vein with a power injector through a 20-gauge intravenous cannula at a rate of $5 \mathrm{ml} / \mathrm{s}$. Four seconds later, another CTP scan was conducted under the following acquisition parameters: $80 \mathrm{kVp}, 150 \mathrm{~mA}, 0.28 \mathrm{~s} /$ rotation, $25 \mathrm{~s}$ CT data acquisition time,
$5 \mathrm{~mm}$ section thickness, field matrix $512 \times 512$ and 40 images per section. The raw data were collected by a radiologist on site. Then, four critical parameters were calculated: regional cerebral blood volume (rCBV), regional cerebral blood flow (rCBF), regional mean transit time ( $\mathrm{rMTT}$ ) and regional time to peak (rTTP).

\section{Description of the Stages of the Pre-Infarction Period}

The pre-infarction period stage of each hemisphere was determined by a radiologist following the same criteria proposed in Gao et al. (19) The primary reference was the normal perfusion area mirrored to the ischaemic area in the contralateral hemisphere. If both hemispheres were ischaemic, the cerebellum was set as a secondary reference. The definition of each pre-infarction stage was listed as follows: Stage 0-normal rTTP, rMTT, rCBV and rCBF; Stage I-delayed rTTP with normal rMTT, rCBF and rCBV (Figure 1A-D); Stage II-delayed rTTP and rMTT with a normal rCBF and a normal or slightly increased rCBV (Figure 2A-D); Stage III-delayed rTTP and $\mathrm{rMTT}$, decreased $\mathrm{rCBF}$ and normal or slightly decreased rCBV (Figure 3A-D) and Stage IV-delayed rTTP and rMTT and decreased rCBF and rCBV (Figure 4A-D). Stages I and II are considered early stages, whereas stages III and IV are late stages. When evaluating individual pre-infarction stages, we used the stage of the more severe hemisphere to represent the individual's hypoperfusion status (25).

\section{Therapeutic Strategies}

There were three types of surgical treatments for MMD: direct

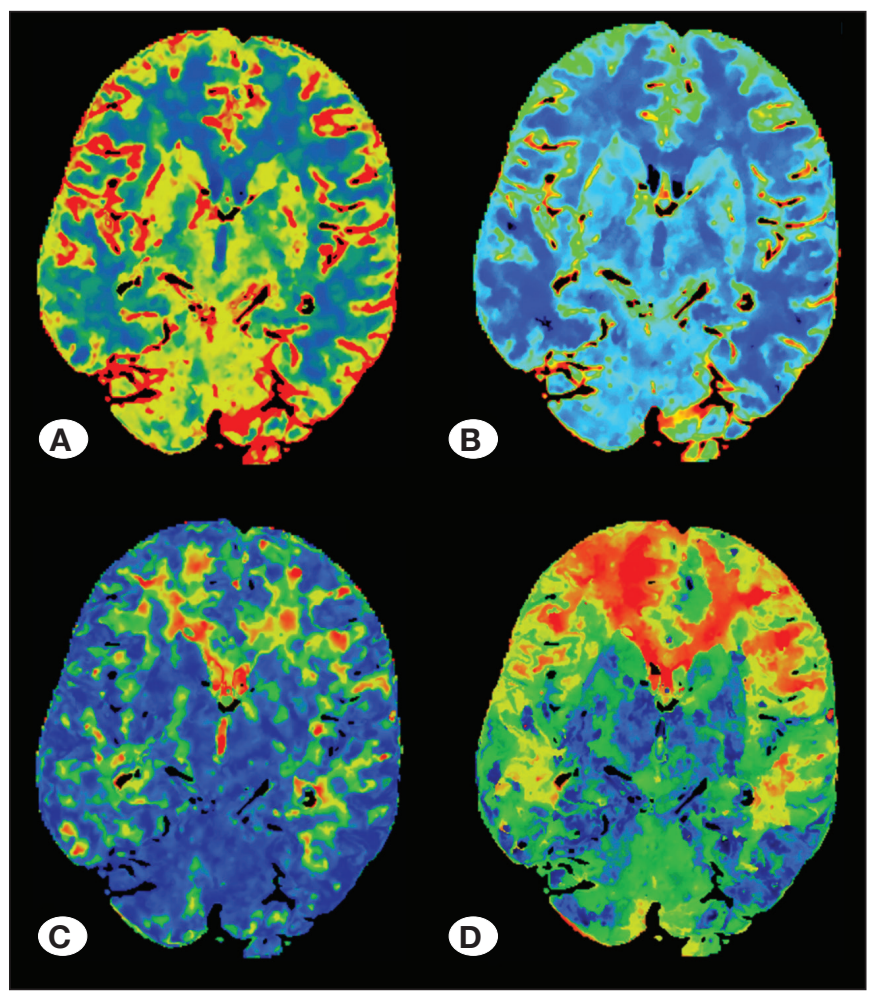

Figure 1: Stage I is shown in the image. The rTTP is delayed and rMTT, rCBF and rCBV are normal in both frontal lobes and both temporal lobes. A) rCBF, B) rCBV, C) rMTT and D) rTTP. 


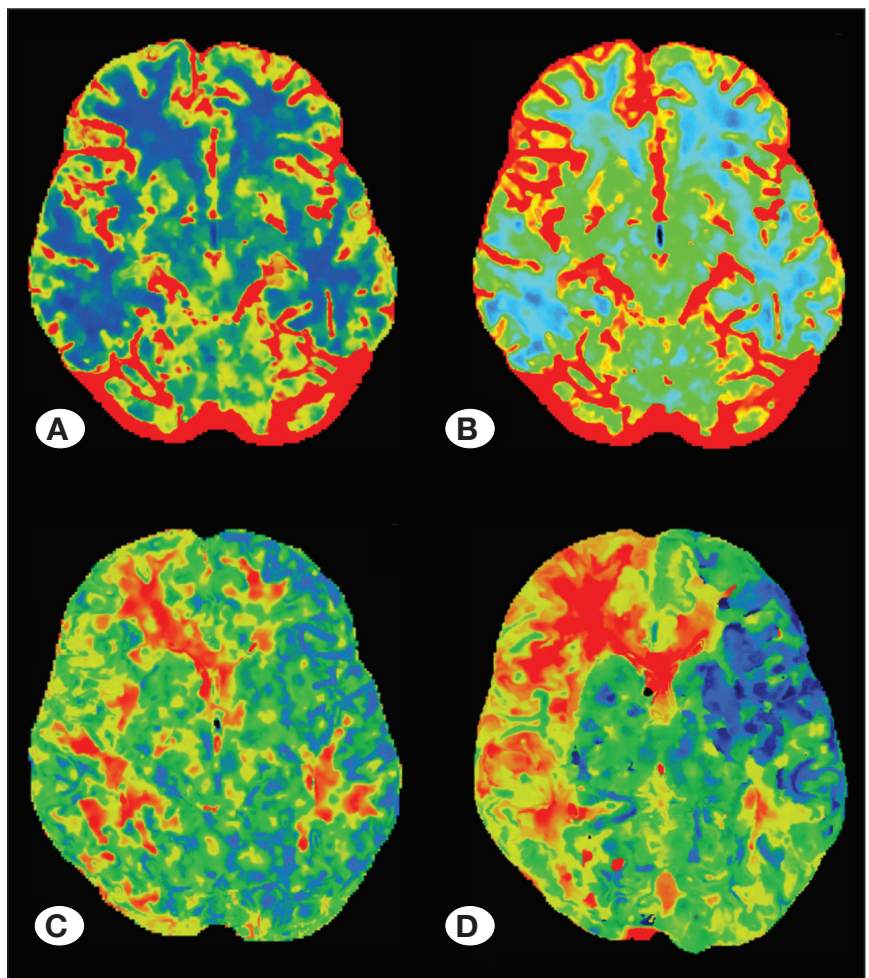

Figure 2: Stage II is shown in the image. The rTTP and rMTT are delayed, and rCBF and rCBV are normal in the right frontal lobe and temporal lobe. A) rCBF, B) rCBV, C) rMTT, D) rTTP.

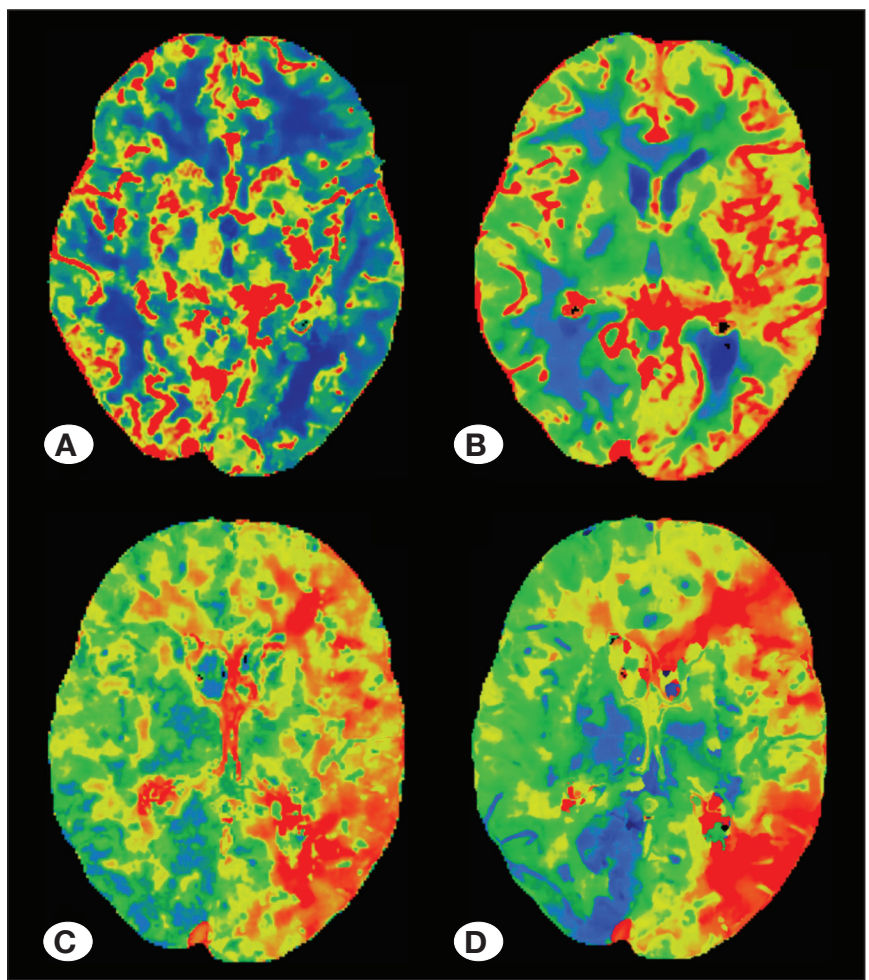

Figure 3: Stage III is shown in the image. The rTTP and rMTT are delayed, rCBF is decreased and rCBV is normal in both frontal lobes and the left temporal lobe. A) rCBF, B) rCBV, C) rMTT, D) rTTP.

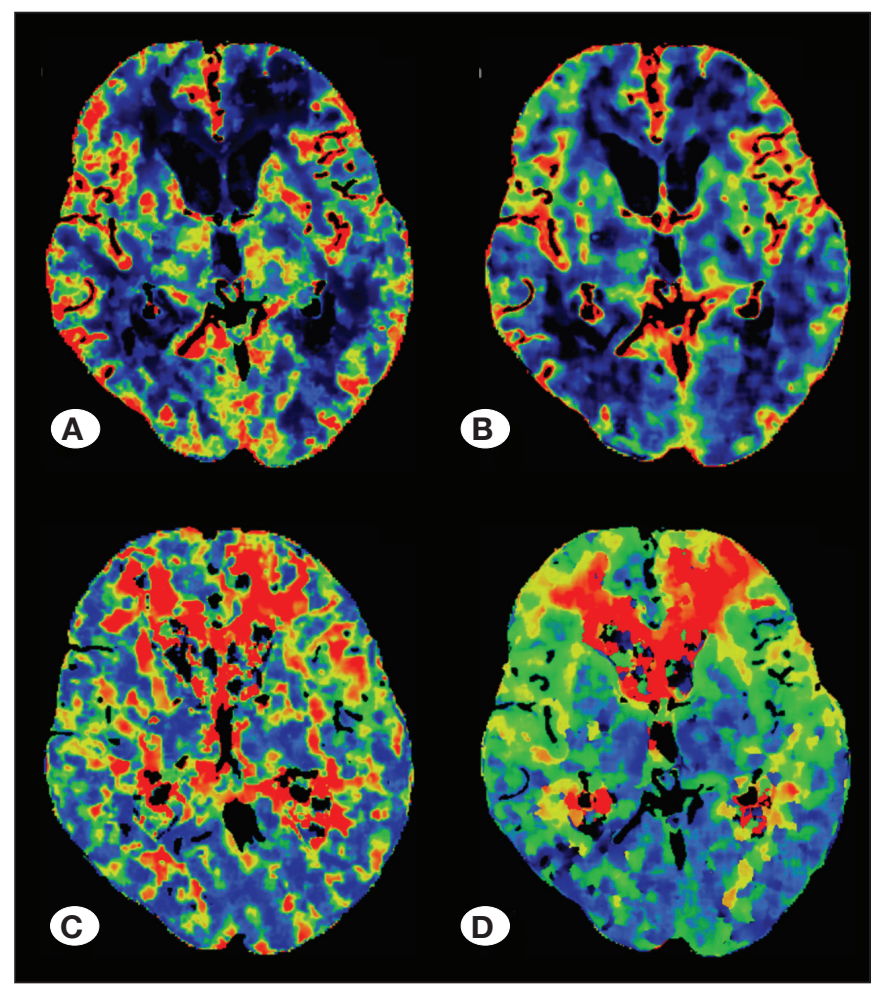

Figure 4: Stage IV is shown in the image. The rTTP and rMTT are delayed, and rCBF and rCBV are decreased in both frontal lobes. A) rCBF, B) rCBV, C) rMTT and D) rTTP.

bypass (DB), indirect bypass (IB) and combined bypass (CB) $(1,4,22)$. In DB surgery, the hypoperfusion status can be alleviated directly after revascularisation by anastomosing the branch of the superficial temporal artery to the cortical branch of the cerebral middle artery. In IB surgery, part of the superficial temporal artery with vascularised tissue is placed on the hypoperfusion cerebral surface or extracranial tissues are in direct contact with the hypoperfusion cerebral surface through multiple drilled bur holes and an incised dura. CB means that DB and IB are simultaneously performed in the same hemisphere during one operation. Surgery is primarily performed on the symptomatic hemisphere to alleviate the hypoperfusion status. If no obvious symptoms are present or symptoms indicate no side-dominance, the hemisphere with a worse perfusion on CTP examination is operated on first. As DB or CB can rectify the hypoperfusion status directly, these two types are the preferred surgical methods for patients with MMD unless the donor or recipient arteries are too slim or fragile for anastomoses. IB is performed instead in these circumstances.

\section{Long-Term Outcomes}

Follow-up was performed at 3 months, 6 months and 1 year after surgery by a clinical visit and telephone interview. The modified Rankin scale (mRS) score was obtained during the follow-up. The ratio of patients with decreased mRS scores in the groups for each stage indicated an improved clinical outcome. 


\section{Statistical Analysis}

Statistical analyses were conducted with SPSS (Windows version 23.0, IBM). Continuous variables were shown as the mean \pm standard deviation, and categorical values were described as percentages (numbers). A chi-squared test was used to compare classified data. Student's $t$-test and Rank sum test were used to compare continuous data. Logistic regression was used to analysis multiple variables. $\mathrm{P}$ value < 0.05 was considered statistical significance. Odds ratios (OR) and $95 \%$ confidence intervals $(\mathrm{Cl})$ were listed after $p$ values.

\section{RESULTS}

We retrospectively studied clinical records of 696 consecutive patients with MMD admitted to Beijing Tiantan Hospital from June 2011 to November 2017. Of them, 195 patients with haemorrhagic MMD and 94 patients with ischaemic MMD

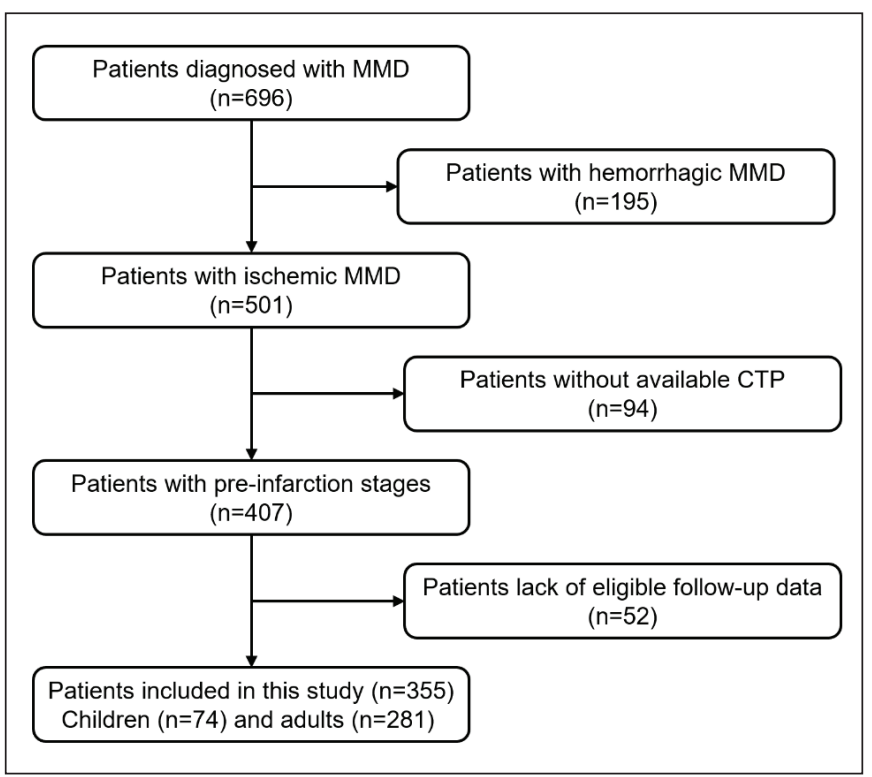

Figure 5: Flow diagram of the study participants. who did not receive a CTP examination were excluded. Fiftytwo patients were excluded due to lack of follow-up data or a follow-up period shorter than 12 months. In the end, a total of 355 patients were involved in this study, including 74 children (age $<18$ years old) and 281 adults (age $\geq 18$ years old; Figure 5). Baseline information is listed in Table I.

In total, 710 hemispheres were classified according to the preinfarction staging system (Table II). In the children's group, 19 (12.9\%) hemispheres were classified as stage 0, $15(10.1 \%)$ hemispheres as stage I, 29 (19.6\%) hemispheres as stage II, 69 (46.6\%) hemispheres as stage III and $16(10.8 \%)$ hemispheres as stage IV. In the adult group, 127 (22.6\%) hemispheres were classified as stage $0,33(5.9 \%)$ hemispheres as stage I, 101 (18.0\%) hemispheres as stage II, 180 (32.0\%) as stage III and $121(21.5 \%)$ hemispheres as stage IV. The proportions of stage 0 and stage IV in the adult group were significantly higher than those in the children's group (stage 0, 22.6\% in the adult group vs. $12.8 \%$ in the children's group, $p=0.009$; stage IV, $21.5 \%$ in the adult group vs. $10.8 \%$ in the children's group, $\mathrm{p}=0.003)$. On the other hand, stage III was more common in the children's group (stage III, $46.6 \%$ in the children's group vs. $32.0 \%$ in the adult group, $p=0.001$ ).

The relationship between patient symptoms and the preinfarction stage is shown in Table III. Patients in stage IV had the highest rate of infarction, patients in stage I showed the lowest rate of infarction, and there was an increasing tendency in the infarction rate from stage I to stage IV. Interestingly the rate of TIA presented a decreasing tendency from stage I to stage IV (Figure 6).

Potential risk factors for clinical outcomes were analysed using logistic regressive analysis (Table IV). Independent risk factors were children/adult group, $\mathrm{mRS}$ on admission and the pre-infarction stage. Other factors, such as symptoms and surgical methods, showed no statistical influence on clinical outcomes. The results also showed that the ratio of improved clinical outcomes of the children's group was much higher than that of the adult group. Moreover, the tendency for clinically improving the ratio from stage I to stage IV varied significantly

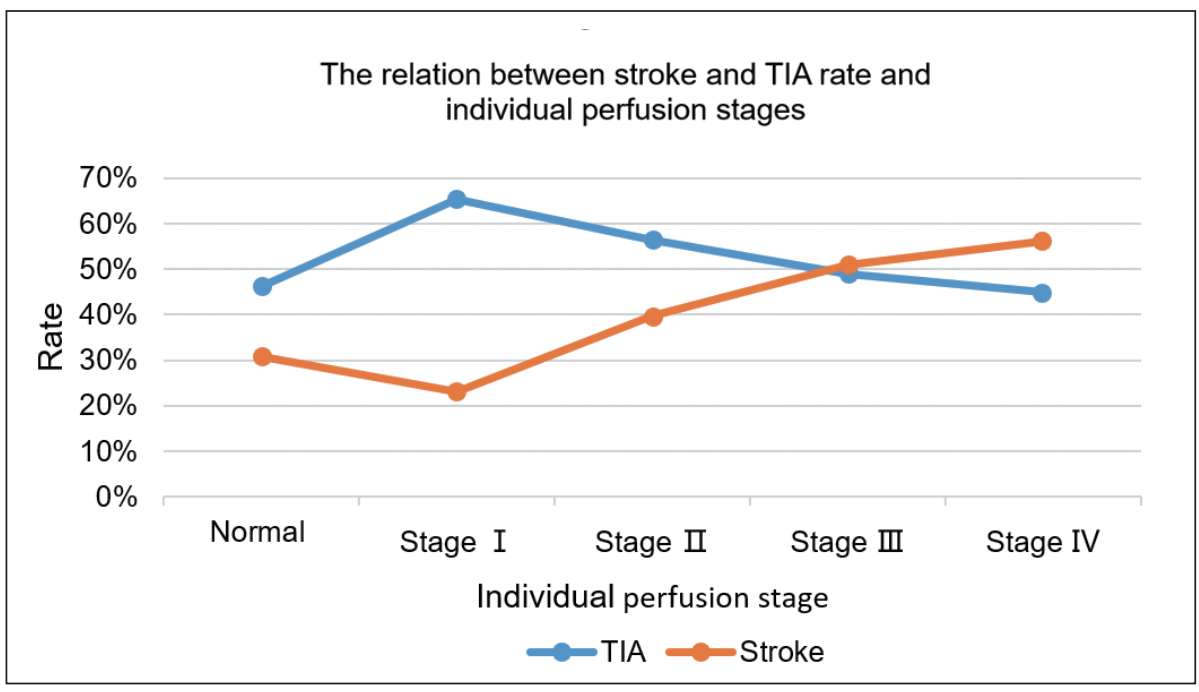

Figure 6: The relation between stroke and TIA rate and individual perfusion stages. 
between the adult group and children's group. In the adult group, patients in stage I had the highest improvement ratio $(66.7 \%)$, and patients in stage IV had the lowest improvement ratio $(44.4 \%)$. There was a decreasing tendency from stage I to stage IV. In the children's group, the improved ratio remained relatively stable (Figure 7 ).
Both the adult and children's group in stage 0 and early stages, including stage I and stage II, had a similar high improvement ratio, and no statistically significant difference was found between adults and children, whether the data was adjusted or not. However, children in stage III and in the late stages, combining stage III and the late stages, received significantly

Table I: Baseline Information of Patients

\begin{tabular}{|c|c|c|c|c|}
\hline & Total patients, $\mathrm{n}=355(\%)$ & Children group, $n=74(\%)$ & Adult group, $n=281(\%)$ & $\mathbf{p}$ \\
\hline Age & & $11.77 \pm 3.22$ & $38.07 \pm 9.82$ & $<0.001$ \\
\hline Gender/male & $169(47.6)$ & 35 (47.3) & $134(47.7)$ & 0.952 \\
\hline \multicolumn{5}{|l|}{ Disease involvement } \\
\hline Unilateral & $33(9.3)$ & $5(6.8)$ & $28(10.0)$ & \\
\hline Bilateral & $322(90.7)$ & $69(93.2)$ & $253(90.0)$ & \\
\hline Symptoms & & & & $<0.001$ \\
\hline Stroke & $167(47.0)$ & $22(29.7)$ & $145(51.6)$ & \\
\hline TIA/frequent TIA & $181(51.0)$ & $46(62.2)$ & $135(48.0)$ & \\
\hline Headache/epilepsy & $87(24.5)$ & $23(31.1)$ & $64(22.8)$ & \\
\hline Suzuki stage & 696 & 148 & 548 & 0.875 \\
\hline 0 & $31(4.5)$ & $5(3.4)$ & $26(4.7)$ & \\
\hline I & $24(3.4)$ & $4(2.7)$ & $20(3.7)$ & \\
\hline II & $63(9.1)$ & $11(7.4)$ & $52(9.5)$ & \\
\hline III & 227 (32.6) & $54(36.5)$ & $173(31.6)$ & \\
\hline IV & $227(32.6)$ & $52(35.1)$ & $175(31.9)$ & \\
\hline $\mathrm{V}$ & $108(15.5)$ & $22(14.9)$ & $86(15.7)$ & \\
\hline VI & $16(2.3)$ & $0(0)$ & $16(2.9)$ & \\
\hline mRS on admission & & & & 0.337 \\
\hline 0 & $7(2.0)$ & $2(2.7)$ & $5(1.8)$ & \\
\hline 1 & $213(60.0)$ & $46(62.2 \%)$ & $167(59.4)$ & \\
\hline 2 & $108(30.4)$ & $24(32.4)$ & $84(29.9)$ & \\
\hline 3 & $19(5.3)$ & $2(2.7)$ & $17(6.1)$ & \\
\hline 4 & $8(2.3)$ & $0(0)$ & $8(2.8)$ & \\
\hline 5 & $0(0)$ & $0(0)$ & $0(0)$ & \\
\hline 6 & $0(0)$ & $0(0)$ & $0(0)$ & \\
\hline Surgerical methods & & & & $<0.001$ \\
\hline $\mathrm{DB}$ & $165(46.5)$ & $19(25.7)$ & $146(52.0)$ & \\
\hline $\mathrm{CB}$ & $70(19.7)$ & $12(16.2)$ & $58(20.6)$ & \\
\hline $\mathrm{IB}$ & $120(33.8)$ & $43(58.1)$ & $77(27.4)$ & \\
\hline Follow-up period(m) & $34.76 \pm 15.47$ & $32.78 \pm 14.75$ & $35.28 \pm 15.63$ & 0.216 \\
\hline
\end{tabular}

Gender ratio was demonstrated by male proportion. Follow-up period was measured by mouth. The letter $n$ represents number and $m$ stands for month. 
Table II: The Difference of CTP Stages between Children Group and Adult Group

\begin{tabular}{|c|c|c|c|c|c|c|}
\hline & $\begin{array}{c}\text { Total patients } \\
(\%)\end{array}$ & $\begin{array}{l}\text { Children group } \\
(\%)\end{array}$ & Adult group (\%) & $\mathbf{p}$ & $95 \% \mathrm{Cl}$ & OR \\
\hline Normal & $146(20.5)$ & $19(12.8)$ & $127(22.6)$ & 0.009 & $0.300 \sim 0.849$ & 0.504 \\
\hline Stage I & $48(6.8)$ & $15(10.1)$ & $33(5.9)$ & 0.066 & $0.954 \sim 3.426$ & 1.808 \\
\hline Stage II & $130(18.3)$ & $29(19.6)$ & $101(18.0)$ & 0.650 & $0.703 \sim 1.761$ & 1.112 \\
\hline
\end{tabular}

Cl stands for confidential interval and OR represents odds ratio.

Table III: The Relationship between TIA \& Stroke and Pre-Infarction Stage

\begin{tabular}{lcccc|cccc}
\hline & \multicolumn{3}{c}{ TIA } & \multicolumn{3}{c}{ Stroke } \\
\hline Normal & $\mathbf{n ~ ( \% )}$ & $\mathbf{p}$ & $\mathbf{9 5 \% \mathbf { C l }}$ & OR & $\mathbf{n}(\%)$ & $\mathbf{p}$ & $\mathbf{9 5 \%}$ CI & OR \\
\hline Stage I & $6(46.2)$ & REF & REF & REF & $4(30.8)$ & REF & REF & REF \\
\hline Stage II & $17(65.4)$ & 0.935 & $0.327 \sim 3.375$ & 1.050 & $6(23.1)$ & 0.097 & $0.099 \sim 1.210$ & 0.347 \\
\hline Stage III & $44(56.4)$ & 0.033 & $1.086 \sim 6.997$ & 2.756 & $31(39.7)$ & 0.005 & $0.086 \sim 0.639$ & 0.234 \\
\hline Stage IV & $73(49.0)$ & 0.140 & $0.860 \sim 2.924$ & 1.585 & $76(51.0)$ & 0.035 & $0.278 \sim 0.954$ & 0.514 \\
\hline
\end{tabular}

The letter $n$ represents number, REF stands for reference and $\mathrm{Cl}$ and OR stands for confidential interval and odds ratio respectively.

Table IV: Potential Risk Factors for Clinical Outcomes Analyzed by Logistic Regressive Analysis

\begin{tabular}{|c|c|c|c|}
\hline & $\mathbf{p}$ & $95 \% \mathrm{Cl}$ & OR \\
\hline Children vs adult & 0.002 & $0.194 \sim 0.701$ & 0.369 \\
\hline \multicolumn{4}{|l|}{ Symptoms } \\
\hline TIA & 0.473 & $0.739 \sim 1.920$ & 1.191 \\
\hline Stroke & 0.126 & $0.404 \sim 1.118$ & 0.672 \\
\hline Headache/epilepsy & 0.224 & $0.808 \sim 2.479$ & 1.415 \\
\hline \multicolumn{4}{|l|}{ Surgerical methods } \\
\hline DB & REF & REF & REF \\
\hline CB & 0.418 & $0.724 \sim 2.181$ & 1.256 \\
\hline IB & 0.459 & $0.656 \sim 2.544$ & 1.292 \\
\hline The mRS on admission & 0.001 & $1.988 \sim 4.533$ & 2.659 \\
\hline \multicolumn{4}{|l|}{ Stage of per-infarction period } \\
\hline Normal & REF & REF & REF \\
\hline Stage I & 0.477 & $0.175 \sim 2.259$ & 0.628 \\
\hline Stage II & 0.025 & $1.154 \sim 8.993$ & 3.222 \\
\hline Stage III & 0.035 & $1.052 \sim 4.172$ & 2.095 \\
\hline
\end{tabular}

$\mathrm{Cl}$ and $\mathrm{OR}$ stands for confidential interval and odds ratio respectively, and REF represents reference. 
Table V: The Differences of Clinical Outcomes between Children Group and Adult Group, before and after Adjusted for Symptoms, Surgical Methods and mRS on Admission

\begin{tabular}{|c|c|c|c|c|c|c|c|c|c|}
\hline & \multirow{2}{*}{$\begin{array}{c}\text { Total } \\
\text { patients } \\
\mathrm{n}(\%)\end{array}$} & \multirow{2}{*}{$\begin{array}{c}\text { Children } \\
\text { groupn } \\
\text { n (\%) }\end{array}$} & \multirow{2}{*}{$\begin{array}{l}\text { Adult } \\
\text { group } \\
\text { n (\%) }\end{array}$} & \multicolumn{3}{|c|}{ Non-adjusted Estimate } & \multicolumn{3}{|c|}{ Adjusted Estimate } \\
\hline & & & & $\mathbf{p}$ & $95 \% \mathrm{Cl}$ & OR & $\mathbf{p}$ & $95 \% \mathrm{Cl}$ & OR \\
\hline Normal & $5(38.5)$ & $1(100)$ & $4(33.3)$ & 0.385 & $0.257 \sim 50.330$ & 3.600 & 0.999 & $0.000 \sim$ & 0.000 \\
\hline Stage I & $19(73.1)$ & $7(87.5)$ & $12(66.7)$ & 0.375 & $0.346 \sim 35.371$ & 3.500 & 0.999 & $0.000 \sim$ & 0.000 \\
\hline Stage II & $52(65.0)$ & $11(68.8)$ & $41(66.1)$ & 0.725 & $0.382 \sim 3.992$ & 1.234 & 0.673 & $0.263 \sim 7.912$ & 1.443 \\
\hline Stage III & $84(56.4)$ & $29(70.7)$ & $55(50.9)$ & 0.029 & $1.077 \sim 5.036$ & 2.329 & 0.016 & $0.151 \sim 0.823$ & 0.353 \\
\hline Stage IV & $41(46.1)$ & $5(62.5)$ & $36(44.4)$ & 0.545 & $0.466 \sim 9.308$ & 2.083 & 0.224 & $0.055 \sim 1.969$ & 0.330 \\
\hline Early Stage & $71(68.3)$ & $18(75.0)$ & $53(66.3)$ & 0.419 & $0.544 \sim 4.296$ & 2.056 & 0.102 & $0.095 \sim 1.239$ & 0.343 \\
\hline Late Stage & $125(52.5)$ & $34(69.4)$ & $91(48.1)$ & 0.008 & $1.248 \sim 4.776$ & 2.441 & 0.004 & $0.164 \sim 0.714$ & 0.342 \\
\hline
\end{tabular}

$\mathrm{Cl}$ and $\mathrm{OR}$ stands for confidential interval and odds ratio respectively.

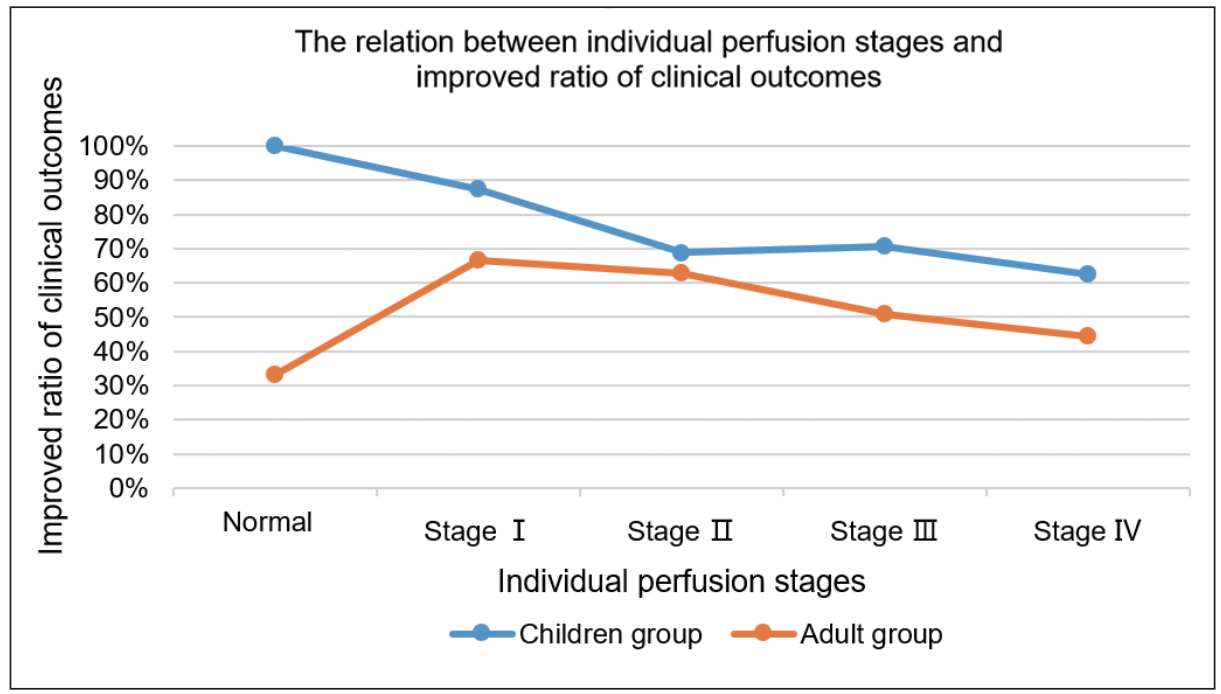

Figure 7: The relation between individual perfusion stages and improved ratios of clinical outcomes. better clinical outcomes than adults (stage III, 70.7\% in the children's group vs. $50.9 \%$ in the adult group, $p=0.029$; late stage, $69.4 \%$ in the children's group vs. $48.1 \%$ in the adult group, $\mathrm{p}=0.008$ ). After the data were adjusted for symptoms, surgical methods and mRS on admission, the children/adult group was an independent factor in stage III and the late stages, and the children's group had better clinical outcomes than the adult group (stage III, OR=0.353, 95\% Cl $0.151-$ $0.823, p=0.016$; late stage, $\mathrm{OR}=0.353,95 \% \mathrm{Cl} 0.164 \sim 0.714$, $\mathrm{p}=0.004$; Table V).

\section{DISCUSSION}

\section{Differences in the Pre-Infarction Period Stages}

In this study, we observed different ischaemic MMD perfusion stage distributions between the adult group and children's group and this, to some extent, supported MMD as a heterogeneous disease $(5,6,10,14,22)$. The results demonstrated that hemispheres in stage III had more in common in the children's group than in the adult group. One possible reason is that, because of children's poor reporting skills, initial symptoms were usually ignored until they became relatively severe. This may explain the high percentage of stage III patients in the children's group and why children always present with obvious ischaemic symptoms at the time of diagnosis $(20,22)$. In adults, MMD could be diagnosed when mild symptoms occurred or through routine examination. Adult patients, therefore, have a better chance of receiving treatment at the early perfusion stages. In this study, we found a higher prevalence of stage 0 in the adult group than in the children's group. On the other hand, if the treatment is delayed, adult patients also have a longer time for the disease to progress, thus, there were more stage IV patients in the adult group.

\section{Long-Term Outcomes}

For long-term outcomes, we found that the pre-infarction staging system correlated with the ratio of improved clinical outcomes in the adult group. Patients in stage I had the highest improved ratio, and patients in stage IV had the lowest 
improved ratio. This result was similar to that of Yin's study (25). However, children with MMD presented a completely different tendency in the improvement rate and had a relatively high improvement rate from stage I to stage IV. Furthermore, children in stage III and stage IV showed a significantly better improvement rate than adults. This supports children having better clinical outcomes $(5,24)$. Our results suggested that children have a great opportunity to improve after active surgical treatment even with severe hypoperfusion before surgery. This result may offer some practical information for clinical practice and surgical strategies.

In this study, three surgical procedures were performed to treat MMD: $C B, D B$ and IB. Adult patients had a higher ratio of $C B$ and DB than child patients. The main factor causing this nonuniform distribution of surgical types is that $\mathrm{DB}$ and $\mathrm{CB}$ require both good donor and recipient arteries, and in some children, these arteries are difficult to find during surgery. Nonetheless, the children's group had better long-term outcomes than adults. Many studies comparing these three procedures demonstrated that CB and DB were not inferior to IB for longterm outcomes $(9,12,15,17)$. Therefore, the different surgery procedures could not explain the different long-term outcomes between adults and children. Moreover, after data adjustment, the children/adult group was an independent factor in stage III and the late stages, and the children's group still had better clinical outcomes than the adult group.

\section{Association between the pre-infarction period stage and pre-} operative symptoms

The stages of cerebral haemodynamic impairment were described previously, and the authors demonstrated that persistent severe decreases in rCBF could increase the risk of stroke $(8,16)$. Similar to their findings, our results showed that patients in stage III and stage IV had a higher ratio of stroke history than patients in stages I and II, illustrating that patients with decreased rCBF have a higher risk of stroke than those without. Furthermore, we found there was a correlation between the stages and history of infarction, with patients in stage I having the lowest rate of infarction and patients in stage IV having the highest rate. This result suggested that patients in the later stages have a higher risk of stroke, and surgical treatments in the early stages could reduce the incidence of stroke.

Patients in stage 0 , however, had a higher rate of stroke in our study than patients in stage I, even though they showed no sign of hypoperfusion. One possible reason is that the total number of patients in stage 0 was small $(n=12)$. This may have caused random errors that led to unreliable results.

\section{CTP and Pre-Infarction Stages}

The feasibility of CTP in detecting haemodynamic impairment and the pathophysiology of cerebral hypoperfusion have been reported in previous studies, and this staging system has been described in detail $(3,7,18)$. In cerebral ischaemia, previous studies found that rTTP was a reliable parameter in terms of detecting perfusion deficiency, and delayed rTTP might be caused by sluggish flow (13). Dilation of the cerebral artery could be the first automatic reflection to maintain the cerebral blood supply. rCBV and rMTT can detect cerebral perfusion autoregulation; therefore increased rCBV and delayed rMTT could be two effective indicators of haemodynamic impairment $(16,18)$. With the progression of ischaemia, collateral blood from the dilated artery could not maintain cerebral perfusion, and rCBF may start to decrease (23). Finally, the compensatory blood volume from dilated collateral arteries could not maintain rCBV and rCBV start to decrease (16). In this method of measurement, the pre-infarction stage was determined by comparing semi-quantitative variables, including rCBF, rCBV, rTTP and rMTT, between abnormal regions and mirrored control regions, but no constant value was defined as the criterion. Despite the above studies supporting this method, further studies are needed to demonstrate its accuracy and reliability.

\section{Limitations}

First, considering the retrospective nature of this study and the heterogeneity of MMD, some research biases are unavoidable, especially when the surgical strategy is determined by surgeons rather than random allocation, and the ratios of the three surgical methods were unable to be fully matched between two groups. Despite this mismatching between two groups barely affecting the conclusion as expounded above, it may reduce the data's potential use in future research. Second, this single-centre study may not represent real-world outcomes and the true current practice of all procedures. The association between clinical outcomes and pre-infarction stages needs to be further verified. Third, the number of patients in stage 0 was relatively small, which may have caused random errors that affected the results. Further studies with larger samples are needed.

\section{CONCLUSION}

There are differences in the perfusion status between children and adult patients with MMD. A pre-infarction staging system is valuable for predicting the outcomes of patients. It has been associated with MMD-related symptoms to some extent. Independent factors were children/adult group, mRS on admission and the pre-infarction stage. Both children and adult patients in the early stages have relatively high improvement rates after surgery, but children had a greater chance of improvement in the later stages. The result may offer some practical information for clinical practice and surgical strategies.

\section{REFERENCES}

1. Acker G, Fekonja L, Vajkoczy P: Surgical management of moyamoya disease. Stroke 49:476-482, 2018

2. Baba T, Houkin K, Kuroda S: Novel epidemiological features of moyamoya disease. J Neurol Neurosurg Psychiatry 79: 900-904, 2008

3. Chen A, Shyr MH, Chen TY, Lai HY, Lin CC, Yen PS: Dynamic CT perfusion imaging with acetazolamide challenge for evaluation of patients with unilateral cerebrovascular stenoocclusive disease. AJNR Am J Neuroradiol 27:1876-1881, 2006 
4. Czabanka M, Pea-Tapia P, Schubert GA, Heppner FL, Vajkoczy $P$ : Proposal for a new grading of moyamoya disease in adult patients. Cerebrovasc Dis 32:41-50, 2011

5. Deng X, Gao F, Zhang D, Zhang Y, Wang R, Wang S, Cao Y, Zhao Y, Pen Y, Ye X, Liu X, Zhang Q, Wang J, Yang Z, Zhao M, Zhao J: Effects of different surgical modalities on the clinical outcome of patients with moyamoya disease: A prospective cohort study. J Neurosurg 128:1327-1337, 2018

6 Duan L, Bao XY, Yang WZ, Shi WC, Li DS, Zhang ZS, Zong R, Han C, Zhao F, Feng J: Moyamoya disease in China: Its clinical features and outcomes. Stroke 43:56-60, 2012

7. Eastwood JD, Alexander MJ, Petrella JR, Provenzale JM: Dynamic CT perfusion imaging with acetazolamide challenge for the preprocedural evaluation of a patient with symptomatic middle cerebral artery occlusive disease. AJNR Am J Neuroradiol 23:285-287, 2002

8. Gordon AL, Goode S, D'Souza O, Auer DP, Munshi SK: Cerebral misery perfusion diagnosed using hypercapnic blood-oxygenation-level-dependent contrast functional magnetic resonance imaging: A case report. J Med Case Rep 4:54-58, 2010

9. Huang Z, Ding X, Men W, Zhang D, Zhao Y, Wang R, Wang $\mathrm{S}$, Zhao J: Clinical features and outcomes in 154 patients with haemorrhagic moyamoya disease: Comparison of conservative treatment and surgical revascularization. Neurol Res 37:886-892, 2015

10. Jea A, Smith ER, Robertson R, Scott RM: Moyamoya syndrome associated with Down syndrome: Outcome after surgical revascularization. Pediatrics 116:e694-701, 2005

11. Kuroda S, Houkin K: Moyamoya disease: Current concepts and future perspectives. Lancet Neurol 7:1056-1066, 2008

12. Kim DS, Huh PW, Kim HS, Kim IS, Choi S, Mok JH, Huh CW: Surgical treatment of moyamoya disease in adults: Combined direct and indirect vs. indirect bypass surgery. Neurol Med Chir (Tokyo) 52:333-338, 2012

13. Koenig M, Kraus M, Theek C, Klotz E, Gehlen W, Heuser L: Quantitative assessment of the ischemic brain bv mean of perfusion-related parameters derived from perfusion CT. Stroke 32:431-437, 2001

14. Liu X, Zhang D, Wang S, Zhao Y, Zhao J: Clinical features and long-term outcomes of moyamoya disease: A single-center experience with 528 cases in China. J Neurosurg 122:392399, 2015
15. Matsushima T, Inoue K, Kawashima M, Inoue T: History of the development of surgical treatments for moyamoya disease. Neurol Med Chir (Tokyo) 52:278-286, 2012

16. Maddula M, Sprigg N, Bath PM, Munshi S: Cerebral misery perfusion due to carotid occlusive disease. Stroke Vasc Neurol 2:88-93, 2017

17. Noh HJ, Kim SJ, Kim JS, Hong SC, Kim KH, Jun P, Bang OY, Chung CS, Lee KH, Lee KH, Kim GM: Long term outcome and predictors of ischemic stroke recurrence in adult moyamoya disease. J Neurol Sci 359:381-388, 2015

18. Ogasawara K, Ogawa A, Yoshimoto T: Cerebrovascular reactivity to acetazolamide and outcome in patients with symptomatic internal carotid or middle cerebral artery occlusion: A xenon-133 single-photon emission computed tomography study. Stroke 33:1857-1862, 2002

19. Gao P, Lin Y: CT perfusion imaging and stages of regional cerebral hypoperfusion in pre-infarction period. Chin J Radiol $37: 882-886,2003$

20. Research Committee on the Pathology and Treatment of Spontaneous Occlusion of the Circle of Willis, Health Labour Sciences Research Grant for Research on Measures for Infractable Diseases: Guidelines for diagnosis and treatment of moyamoya disease (spontaneous occlusion of the circle of Willis). Neurol Med Chir (Tokyo) 52:245-266, 2012

21. Suzuki J, Takaku A: Cerebrovascular moyamoya disease: Disease showing abnormal net-like vessels in base of brain. Arch Neurol 20:288-299, 1969

22. Scott RM, Smith ER: Moyamoya disease and moyamoya syndrome. N Engl J Med 360:1226-1237, 2009

23. Togao O, Mihara F, Yoshiura T, Tanaka A, Noguchi T, Kuwabara Y, Kaneko K, Matsushima T, Honda H: Cerebral hemodynamics in moyamoya disease: Correlation between perfusion-weighted MR imaging and cerebral angiography. AJNR Am J Neuroradiol 27:391-397, 2006

24. Ueki K, Meyer FB, Mellinger JF: Moyamoya disease: The disorder and surgical treatment. Mayo Clin Proc 69:749-757, 1994

25. Yin $\mathrm{H}$, Liu X, Zhang D, Zhang $Y$, Zhao J: A novel staging system to evaluate cerebral hypo-perfusion in patients with moyamoya disease. Stroke 49:2837-2843, 2018 